\title{
Insight into the Ebola virus nucleocapsid assembly mechanism: crystal structure of Ebola virus nucleoprotein core domain at $1.8 \AA$ resolution
}

\author{
Shishang Dong ${ }^{1,2,3}$, Peng Yang ${ }^{1,3}$, Guobang $\mathrm{Li}^{1,3}$, Baocheng Liü ${ }^{2,3}$, Wenming Wang ${ }^{2,3}$, Xiang Liu ${ }^{3}$, \\ Boran Xia ${ }^{3}$, Cheng Yang ${ }^{1,3}$, Zhiyong Lou ${ }^{1}$, Yu Guo ${ }^{1,3 \bowtie}$, Zihe Rao ${ }^{1,2,3 \bowtie}$ \\ ${ }^{1}$ State Key Laboratory of Medicinal Chemical Biology and College of Pharmacy, Nankai University, Tianjin 300071, China \\ ${ }^{2}$ College of Life Sciences, Nankai University, Tianjin 300071, China \\ ${ }^{3}$ Tianjin International Joint Academy of Biotechnology \& Medicine, Tianjin 300457, China \\ $\bowtie$ Correspondence: guoyu@nankai.edu.cn (Y. Guo), raozh@nankai.edu.cn (Z. Rao) \\ Received February 13, 2015 Accepted April 14, 2015
}

\begin{abstract}
Ebola virus (EBOV) is a key member of Filoviridae family and causes severe human infectious diseases with high morbidity and mortality. As a typical negative-sense single-stranded RNA (-ssRNA) viruses, EBOV possess a nucleocapsid protein (NP) to facilitate genomic RNA encapsidation to form viral ribonucleoprotein complex (RNP) together with genome RNA and polymerase, which plays the most essential role in virus proliferation cycle. However, the mechanism of EBOV RNP formation remains unclear. In this work, we solved the high resolution structure of core domain of EBOV NP. The polypeptide of EBOV NP core domain ( $\mathrm{NP}_{\text {core }}$ ) possesses an $\mathrm{N}$-lobe and $\mathrm{C}$-lobe to clamp a RNA binding groove, presenting similarities with the structures of the other reported viral NPs encoded by the members from Mononegavirales order. Most strikingly, a hydrophobic pocket at the surface of the C-lobe is occupied by an $\alpha$ -

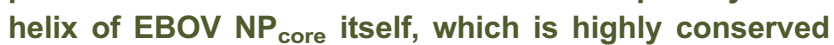
among filoviridae family. Combined with other biochemical and biophysical evidences, our results provides great potential for understanding the mechanism underlying EBOV RNP formation via the mobility of EBOV NP element and enables the development of antiviral therapies targeting EBOV RNP formation.
\end{abstract}

KEYWORDS Filoviridae, Ebola virus, nucleoprotein, nucleocapsid, crystal structure, assembly mechanism

\section{INTRODUCTION}

The members of filovirus family, including Ebola virus (EBOV) and Marburg virus (MARV) and Lloviu virus (LLOV) (Kuhn et al., 2010), cause highly lethal hemorrhagic fever in human beings with extremely high morbidity and mortality. EBOV is typically found in Central Africa, but re-emerged in Western Africa in 2014 to cause a worldwide-spreading outbreak spreading. Till April 2015, 25,591 suspected cases and 10,602 dead cases were reported (http://www.cdc.gov/ vhf/ebola/outbreaks/2014-west-africa/case-counts.html). Although several chemical agents, antibodies and vaccines are found to inhibit EBOV in animal or human, there is no therapeutics with high efficacy can be provided for clinical usage.

EBOV is a typical non-segmented negative-sense singlestranded RNA (-ssRNA) virus (Muhlberger et al., 1999). The single-stranded RNA genome of EBOV encodes the surface glycoprotein (GP), an RNA-dependent RNA polymerase $(\mathrm{RdRp})$, a nucleocapsid protein (NP), as well as viral protein (VP)35, VP40, VP30 and VP24 (Muhlberger et al., 1999). Similar to other -ssRNA viruses, the RNA genome of EBOV cannot exist as a naked form, but must be encapsidated by $\mathrm{NP}$ and further form a ribonucleoprotein (RNP) complex together with RdRp (Sun et al., 2012; Zhou et al., 2013). After entry into the cytoplasm through membrane fusion mediated by glycoproteins, the RNP is released from the virion and serves as the template with which the copackaged RdRp transcribes mRNAs from the viral genome in the RNP. In the later stage of virus replication, complementary positive- 
strand RNA (cRNA) is produced in the form of an RNP. The RNP serves as the template for replication that generates viral genomic RNA in the form of an RNP ready to be packaged in the virion. Throughout the entire virus replication cycle of a -ssRNA virus, the genome length viral RNA (cRNA or viral genomic RNA) is only present in the form of an RNP that either serves as a template for RNA synthesis or is packaged in the virion. Therefore, the formation and correct function of RNP is essential for -ssRNA virus proliferation (Sun et al., 2012; Zhou et al., 2013; Ruigrok et al., 2011).

Structural knowledge of -ssRNA virus RNPs was initiated by studying non-segmented -ssRNA viruses, including Borna disease virus (BDV) (Rudolph et al., 2003), rabies virus (Albertini et al., 2006), vesicular stomatitis virus (VSV) (Green et al., 2006), and respiratory syncytial virus (RSV) (Tawar et al., 2009), and this knowledge has been greatly enhanced by recent investigations of segmented -sSRNA viruses, including arenaviruses (Hastie et al., 2011a, b; Qi et al., 2011), bunyaviruses (Dong et al., 2013; Ferron et al., 2011; Guo et al., 2012; Jiao et al., 2013; Li et al., 2013; Niu et al., 2013; Raymond et al., 2010), and influenza virus ( $\mathrm{Ng}$ et al., 2008; Ye et al., 2006; Arranz et al., 2013; Chenavas et al., 2013; Moeller et al., 2013). Furthermore, advances in the visualization of native or authentic RNP through electron microscopy led to the understanding of the dynamic processes of RNP formation at molecular level. In particular, recent results have clearly demonstrated that viral NP could be directly used as the target for antiviral development (Gerritz et al., 2011; Kao et al., 2010), raising a great potential to find new antiviral agents with novel mechanism against drug resistance occurred in traditional antiviral drugs. Recent work on cryo-electron microscopy and tomography help us visualize Filoviridae virus particles and formation of nucleocapsid (Bharat et al., 2012; Bharat et al., 2011). However, the structural details of EBOV NP and the molecular mechanism of EBOV RNP formation are largely unknown. The exclusive structural information is that a recent work identified the boundaries of $\mathrm{N}$ - and $\mathrm{C}$-terminal domains of EBOV NP and solve the crystal structure of the C-terminal domain spanning residues 641-739 (Dziubanska et al., 2014). However, the structure of C-terminal domain of EBOV NP did not hint to the biological function of this region. All of these promote us to initiate the biochemical and structural analysis on EBOV NP to achieve the understanding of the mechanism of EBOV RNP formation and explore the potential drug target for the discovery of anti-EBOV agents.

\section{RESULTS}

Biochemical analysis of the recombinant core domain of EBOV NP

Previous studies have demonstrated that $\mathrm{N}$-terminal truncation of EBOV NP resulted the loss-of-function in NP-NP interaction and the first 450 amino-acid of EBOV NP, forming the core domain, is sufficient for the formation of nucleocapsid-like structures and viral genome replication (Watanabe et al., 2006). However, most of previous expression and purification work aimed for structural investigation failed because of the tendency to oligomerization and precipitate. To screen the best construct suitable for the crystallographic work on the core domain of EBOV NP, we generate a series of truncation and mutation constructs. All constructs containing the first 30 amino acids of EBOV NP result to high tendency to oligomerize and precipitate, even in ultra-high ion strength condition.

The best construct with the highest expression yield and biochemical features covers the region of residue 36-351 of

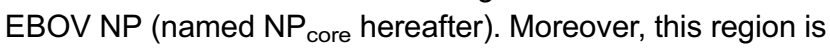
highly conserved not only in different strains of EBOV, but also in Marburg virus and Lloviu virus, another two representative member in Filoviridae family. In particular, this region was revealed to exert the both the function of RNA binding and further NP oligomerization to form high-order RNP. We therefore performed further crystallographic and biochemical investigation on this $\mathrm{NP}_{\text {core }}$ region.

Because binding RNA and oligomerization are two key features for virally encoded NP, we further analyzed the

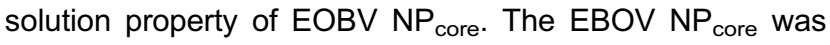
expressed in Escherichia coli and purified under physiological conditions. The retention volume of the recombinant EBOV $\mathrm{NP}_{\text {core }}$ protein in size-exclusion chromatography peaks at $16 \mathrm{~mL}$ (Fig. 1A), corresponding to a molecular weight to $35 \mathrm{kDa}$. SDS-PAGE analysis indicated that the major peaks contained a protein with expected size for EBOV NP core, while the $A_{280} / A_{260} U V$ absorption ratio of 1.6 demonstrated that there was no nucleic acid binding to

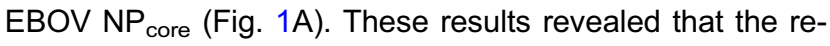
combinant EBOV NP core exists as a monomeric state in solution and do not contain nucleic acids acquired from expression host cells.

\section{Overall structure of EBOV $\mathrm{NP}_{\text {core }}$}

The EBOV NP core was successfully crystallized, and the crystal structure was subsequently determined using the single-wavelength anomalous dispersion (SAD) method and refined to a high resolution of $1.8 \AA$ with a final $R_{\text {work }}$ of $19.2 \%\left(R_{\text {free }}=22.3 \%\right)($ Table 1$)$.

In the structure of EBOV NP core, one molecule is observed in the crystallographic asymmetric unit. Further analysis suggests that the intramolecular interactions are associated with crystal packing and the monomer should be the biological unit. All residues of EBOV NP core polypeptide were be built into the final model, except three short gaps (residues A123-S126, E139-T143, K257-R263), and one loop region covering A326-V334 cannot be traced due to the lack of interpretable electron density, indicating their intrinsic structural flexibility (Fig. 1B).

The structure of EBOV $\mathrm{NP}_{\text {core }}$ presents a novel protein folding within viral NP family and displays a compact architecture with dimensions approximately of $53 \AA \times 40 \AA \times 75 \AA$. 
A

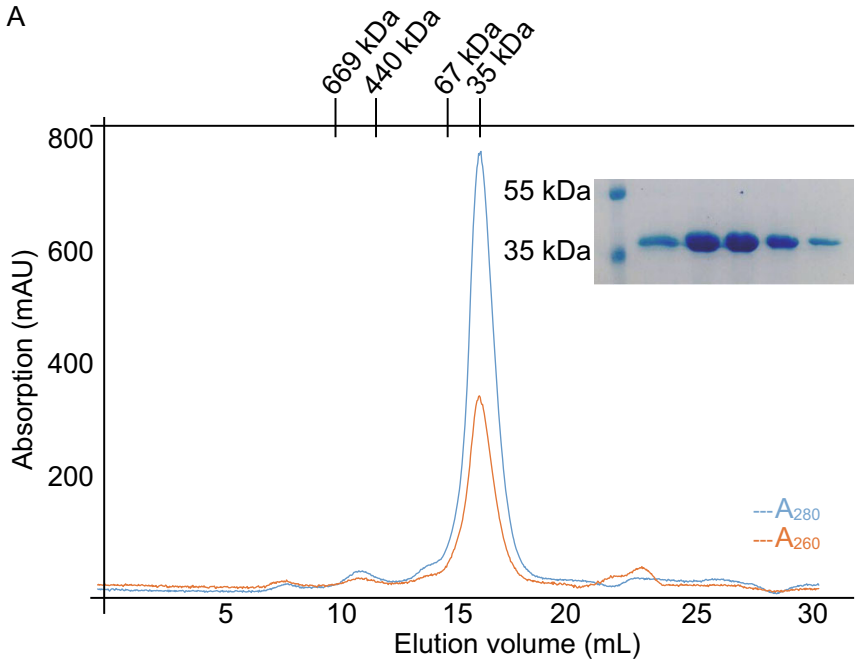

B

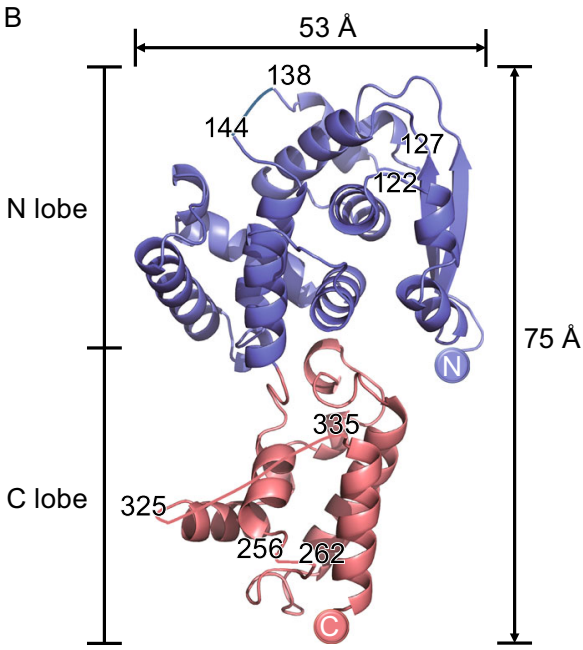

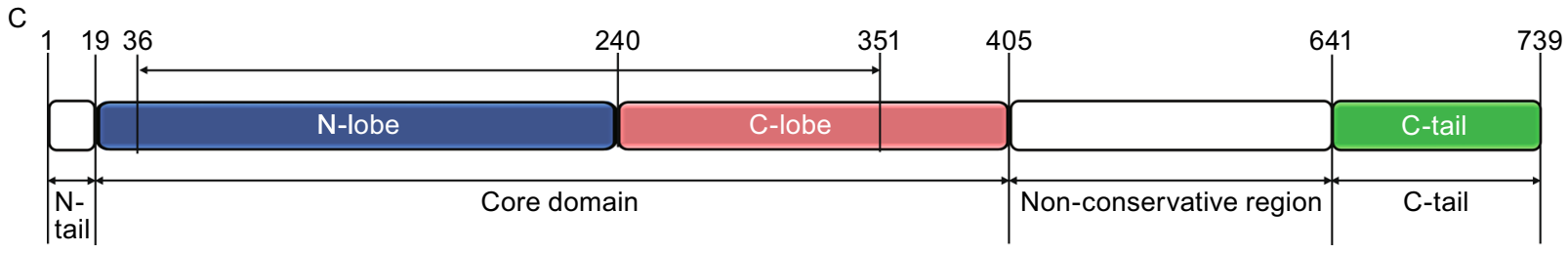

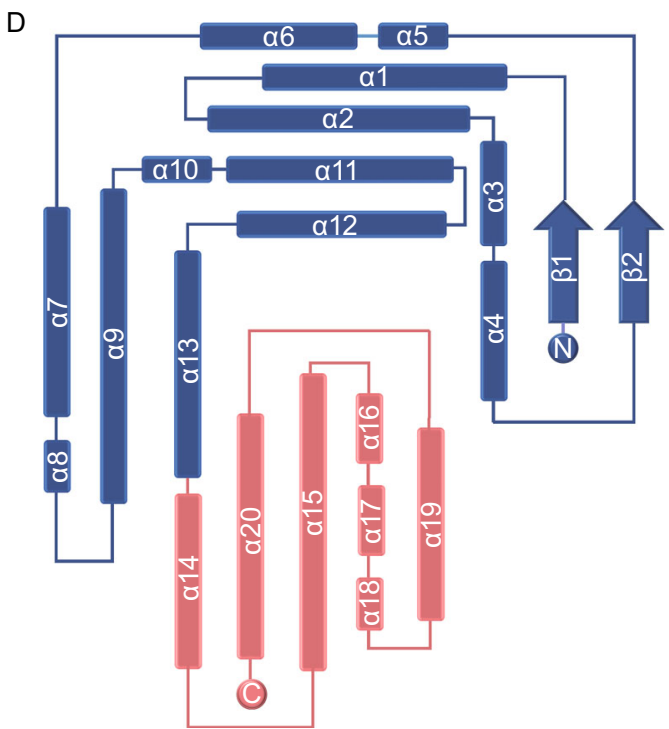

Figure 1. Purification and structure of EBOV NP core. (A) Size exclusion chromatography (SEC) of EBOV NP containing EBOV NP core was injected into a Superdex-200 column. The molecular weights of standard protein markers are shown on the top. Blue and red lines denote $A_{280}$ and $A_{260}$, respectively. SDS-PAGE analysis of the peak fractions is shown inset. (B) Overall structure of EBOV NP core. The polypeptide of EBOV NP core is shown as colored cartoon. The $\mathrm{N}$-lobe and C-lobe are colored as blue and red, respectively. Missing residues are linked by dotted lines. (C) Schematic diagram of the domain organization in the primary sequence of EBOV NP. N-lobe, C-lobe, and C-tail are colored as slate blue, salmon red, and green, respectively. N-tail and nonconservative region are colored as blank. (D) Topology diagram of EBOV NP $\mathrm{P}_{\text {core }}$ helices are presented as rectangulars and strands are shown as arrows. The color scheme is the same as that in (B) and (C).

EBOV NP$P_{\text {core }}$ is featured by two relatively separate portions, an N-lobe (V36-R240), and a C-lobe (F241-E351) (Fig. 1B and $1 \mathrm{C})$. Both two domains are predominantly composed of $\alpha$-helices, in which the $\mathrm{N}$-lobe consisted of thirteen $\alpha$-helices and two $\beta$-strands and the C-lobe consisted of seven $\alpha$-helices (Fig. 1B and 1D). Similar with most virus-encoded NPs, 
Table 1. Data collection and refinement statistics

\begin{tabular}{|c|c|c|}
\hline Parameters & Native & SeMet \\
\hline \multicolumn{3}{|l|}{ Data collection statistics } \\
\hline \multicolumn{3}{|l|}{ Cell parameters } \\
\hline$a(\AA)$ & 59.8 & 59.3 \\
\hline$b(\AA)$ & 162.9 & 162.9 \\
\hline$c(\AA)$ & 31.3 & 31.1 \\
\hline$\alpha, \beta, \gamma\left(^{\circ}\right)$ & $90.0,90.0,90.0$ & $90.0,90.0,90.0$ \\
\hline Space group & $P 2{ }_{1}{ }_{1} 2$ & $P 2{ }_{1} 2{ }_{1}$ \\
\hline Wavelength used $(\AA)$ & 0.9785 & 0.9785 \\
\hline Resolution $(\AA)$ & $40.7-1.79(1.86-1.79)^{\mathrm{c}}$ & $40.1-2.4(2.49-2.40)$ \\
\hline No. of all reflections & $312,740(14,770)$ & $158,723(7888)$ \\
\hline No. of unique reflections & $29,588(1477)$ & $12,497(580)$ \\
\hline Completeness (\%) & $99.16(93.53)$ & $99.50(96.14)$ \\
\hline Average $/ / \sigma(I)$ & $20.04(3.94)$ & $34.08(8.9)$ \\
\hline$R_{\text {merge }}^{\mathrm{a}}(\%)$ & $8.4(54.6)$ & $16.6(45.1)$ \\
\hline \multicolumn{3}{|l|}{ Refinement statistics } \\
\hline No. of reflections used $(\sigma(F)>0)$ & 29492 & \\
\hline$R_{\text {work }}^{\mathrm{b}}(\%)$ & 19.19 & \\
\hline$R_{\text {free }}^{\mathrm{b}}(\%)$ & 22.30 & \\
\hline r.m.s.d. bond distance $(\AA)$ & 0.011 & \\
\hline r.m.s.d. bond angle $\left({ }^{\circ}\right)$ & 1.19 & \\
\hline Average $B$-value $\left(\AA^{2}\right)$ & 39.5 & \\
\hline No. of protein atoms & 2512 & \\
\hline No. of ligand atoms & 0 & \\
\hline No. of solvent atoms & 293 & \\
\hline \multicolumn{3}{|l|}{ Ramachandran plot } \\
\hline Res. in favored regions (\%) & 98.23 & \\
\hline Res. in generously allowed regions (\%) & 1.77 & \\
\hline Res. in disallowed regions (\%) & 0 & \\
\hline
\end{tabular}

${ }^{\text {a }} R_{\text {merge }}=\Sigma_{\mathrm{h}} \Sigma_{1}\left|l_{\text {ih }}-<l_{\mathrm{h}}\right\rangle\left|/ \Sigma_{\mathrm{h}} \Sigma_{1}<l_{\mathrm{h}}\right\rangle$, where $\left.<l_{\mathrm{h}}\right\rangle$ is the mean of the observations $l_{\text {in }}$ of reflection $\mathrm{h}$.

b $R_{\text {work }}=\Sigma\left(|| F_{\mathrm{p}}(\mathrm{obs})|-| F_{\mathrm{p}}(\right.$ calc $\left.)||\right) / \Sigma\left|F_{\mathrm{p}}(\mathrm{obs})\right| ; R_{\text {free }}$ is an $R$ factor for a pre-selected subset $(5 \%)$ of reflections that was not included in refinement.

${ }^{c}$ Numbers in parentheses are corresponding values for the highest resolution shell.

the $\mathrm{N}$ - and $\mathrm{C}$-lobes clamp a highly positively charged groove to encapsidate viral RNA.

\section{The RNA binding groove of EBOV NP}

The $A_{280} / A_{260}$ ratio of EBOV NP core is approximate 1.6 and indicates no RNA binding occurred during the expression

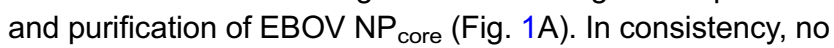
continuous nucleic acids electron density could be observed on the surface of EBOV NP core.

However, highly positively charged pockets on the molecular surface of EBOV NP core coordinated the investigation on the RNA binding site (Fig. 2). There are two positivecharged regions located in the structure of EBOV NP ${ }_{\text {core }}$, a major one and a minor one. The major positive-charged crevice is located in the interface of $\mathrm{N}$-lobe and $\mathrm{C}$-lobe, in which $\mathrm{K} 160, \mathrm{~K} 171, \mathrm{R} 174$, and $\mathrm{K} 248$ contribute the most essential positive charge. An additional minor positivecharged region is adjacent to the major crevice and is consisted by R205, K211, and R298. Positive-charged residues, including lysine, arginine, and histidine residues, are known to interact with the ribose and phosphate moieties of nucleic acid and thus dominate the RNA encapsidation process of viral NPs (Albertini et al., 2006; Green et al., 2006; Hastie 

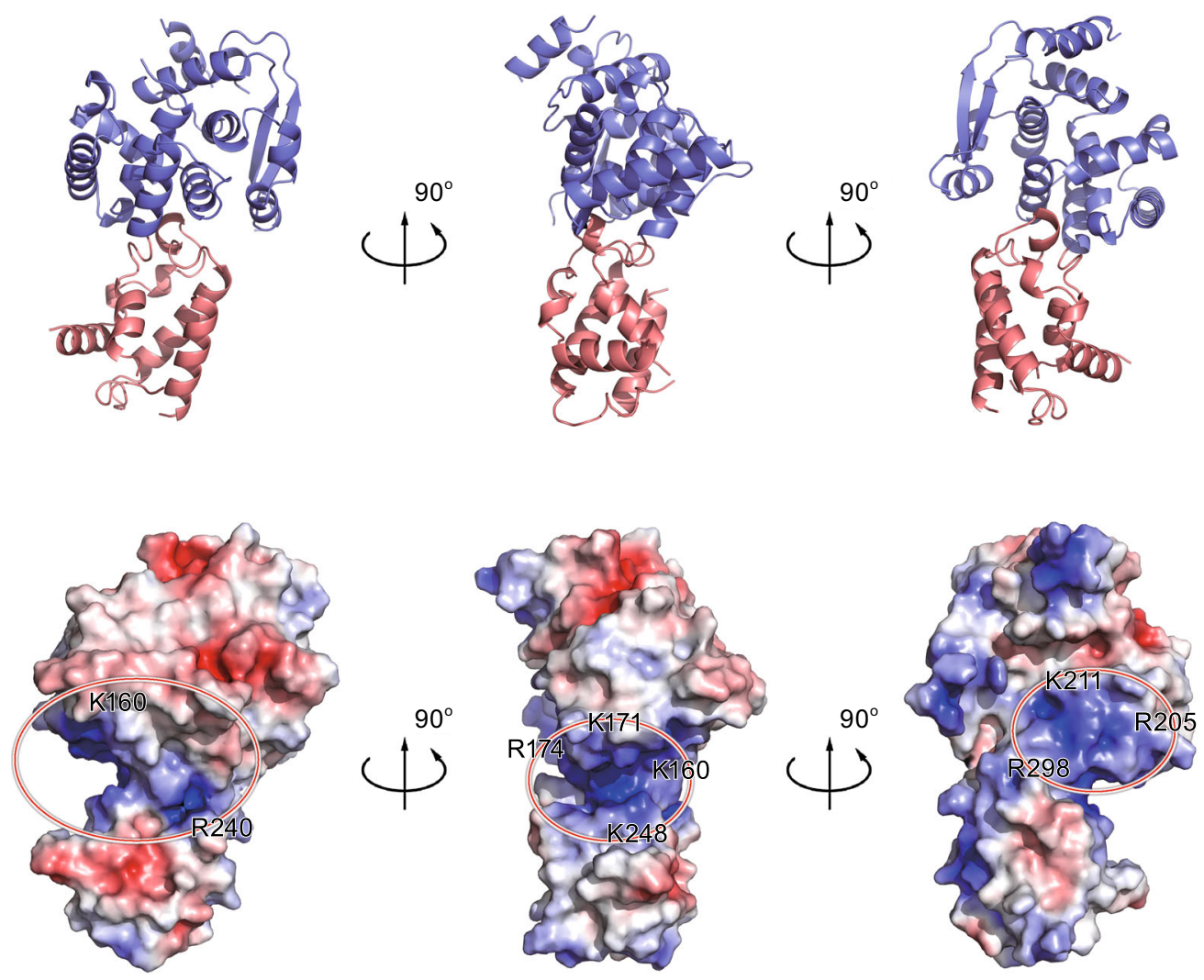

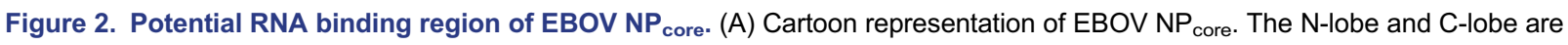
colored as slate blue and salmon red, respectively. (B) Electrostatic surface potential of EBOV NP core. The electrostatic surface

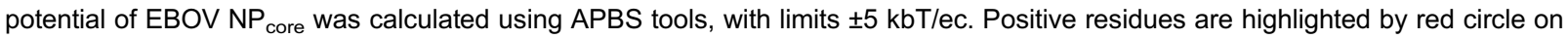
EBOV NP core, suggesting the presence of several positively charged grooves that may be involved in RNA binding.

et al., 2011; Guo et al., 2012; Li et al., 2013; Ariza et al., 2013; Raymond et al., 2012; Reguera et al., 2013). To be consistent with this structural observation in the major groove in $\mathrm{EBOV} \mathrm{NP}_{\text {core, }}$, previous works have demonstrated that deletion of these basic residues or substitutions to alanine residues significantly impaired the RNA binding affinity of EBOV NP or EBOV replication (Muhlberger et al., 1999; Watanabe et al., 2006; Noda et al., 2010; Huang et al., 2002; Leung et al., 2015). Taken together, these structural and biochemical evidences designated that the RNA binding groove of EBOV NP is located in the interface of $\mathrm{N}$ - and $\mathrm{C}$-lobes and demonstrated the essential role of the key basic residues for RNA binding and EBOV proliferation.

\section{Structural comparison of EBOV NP core with other NPs in Mononegavirales}

DALI (Holm and Rosenstrom, 2010) and SSM (Krissinel and Henrick, 2004) analysis revealed that EBOV NP core $_{\text {presents }}$ structural similarity with viral NPs encoded by the members of Mononegavirales order, including RSV (Tawar et al.,
2009), parainfluenza virus 5 (PIV-5) (PDB code: $4 \mathrm{XJN}$ ), and Nipah virus (NiV) (Yabukarski et al., 2014), and etc. Alignment of EBOV NP core with RSV NP structures gives an overall root-mean-square deviation (r.m.s.d.) of $4.4 \AA$ for all $\mathrm{C} \alpha$ atoms of the 375 aligned residues, while the alignment with PIV5 NP gives an r.m.s.d. of $4.2 \AA$ for 395 residues and NiV NP gives an r.m.s.d. of $3.6 \AA$ for 330 residues (Fig. $3 A$ ).

Not only NPs of RSV, PIV-5, and NiV, the overall structure of all Mononegavirales NPs shows similar topology, despite of low primary sequence homology. All of these NPs include an N-lobe and a C-lobe to clamp the RNA binding site on interface. However, the formations of RNP achieved by viral NPs are variable. The most striking difference is that the continuous nucleic acid chains are found to be bound in the inner side of NP-RNA particles of rabies virus (Albertini et al., 2006) and VSV (Green et al., 2006), but the bound RNAs are exposed to the outer side of NP-RNA particles of RSV (Tawar et al., 2009) and PIV5 NP. According to the structure

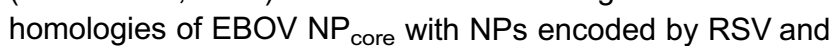
PIV5, we speculate the RNA binding position of EBOV NP should adopt same pattern. Notably, besides the core 


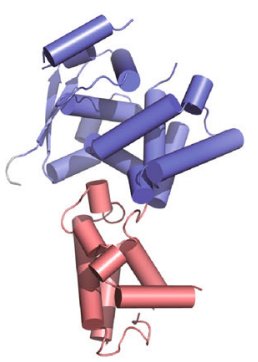

EBOV NP core

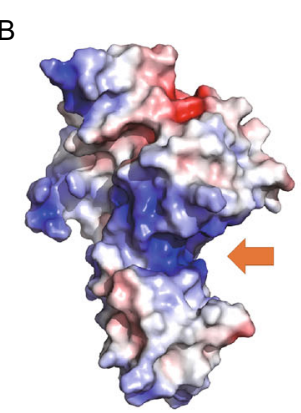

EBOV NP $\mathrm{P}_{\text {core }}$

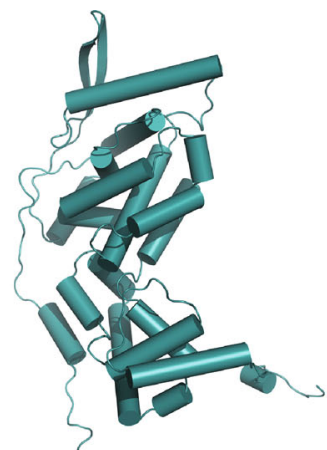

RSV NP PDB code:2WJ8 r.m.s.d $=4.4$ Z-score $=13.2$ Nres $=375$

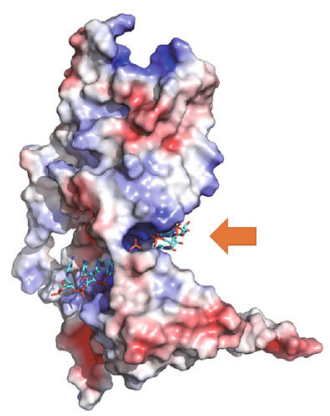

RSV NP

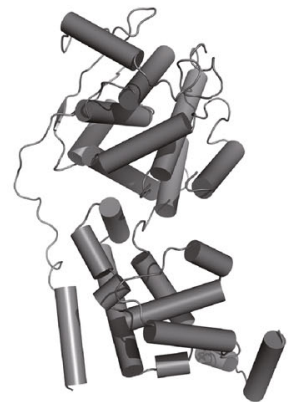

PIV5 NP PDB code:4XJN r.m.s.d $=4.2$ Z-score $=15.7$

Nres $=395$

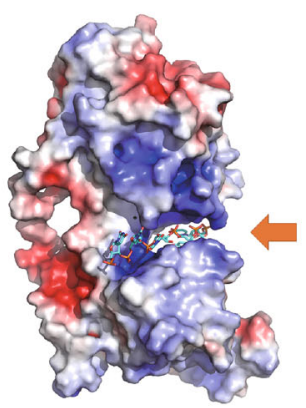

PIV5 NP

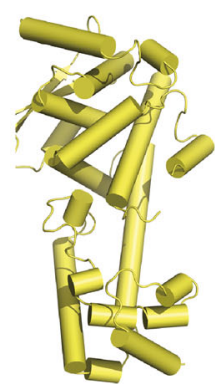

NiV NP PDB code:4CO6 r.m.s.d $=3.6$ Z-score $=15.7$ Nres $=330$

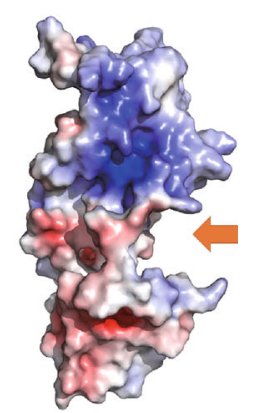

NiV NP

Figure 3. Structural comparison of NPs from Mononegavirales viruses. (A) Structural comparison of EBOV NP core with RSV, PIV5, and NiV NP. NPs are displayed as cartoon. RSV (PDB code: 2WJ8), PIV5 (PDB code: 4XJN), and NiV (PDB code: 4CO6) NPs are colored cyan, gray, and yellow, respectively. Alignment information is listed under each molecule. All molecules are aligned to the structure of EBOV NP core and shown in the same orientation. (B) Electrostatic potential comparison of EBOV NP core with RSV, PIV5, and NiV NP. Positively charged pockets for RNA binding are indicated with orange arrows. RNA molecules in RSV and PIV5 NPs are shown as colored sticks.

domain, EBOV NP accommodates an non-conservative region and a C-terminal tail, which is nearly 200 amino-acid length, suggesting EBOV NP may have additional biological functions beyond RNA binding and oligomerization to form high-ordered RNP. Actually, a breakthrough in the understanding of virally encoded NP is that Lassa fever virus (LASV) (Hastie et al., 2011; Qi et al., 2011) and CrimeanCongo hemorrhagic fever (CCHFV) (Guo et al., 2012) NPs do have an enzymatic activity. These indicate that the further analysis on the extension parts of EBOV NP is necessary to dissect its precise biological roles.

Helix-20 in EBOV $\mathrm{NP}_{\text {core }}$ indicates an essential hydrophobic pocket

A critical step for -ssRNA RNP function is that NP releases the encapsidated RNAs and transfers RNAs to the catalytic center of RdRp for polymerase reaction. Recent works on VSV (Green and Luo, 2009), NiV (Yabukarski et al., 2014), and arenavirus (Kranzusch and Whelan, 2011) have revealed several key co-factors or chaperones to regulate this key process. For example, a phosphoprotein $(P)$ was shown to be the key regulator to VSV RNP formation and the $\mathrm{C}$-terminal domain of $\mathrm{P}$ binds primarily to the C-lobe of VSV NP within NP-RNA particles (Green and Luo, 2009). Moreover, in the structure of NiV NP-P complex, a peptide derived from $P$ was determined to locate at one hydrophobic crevice, protecting host cells against viral replication by inhibiting viral RNP formation (Yabukarski et al., 2014). Interestingly, the superimposition of EBOV NP core with NiV NP-P complex suggests a similar hydrophobic pocket (Fig. 4A). This hydrophobic pocket lies on the C-lobe of EBOV NP core, which is adjacent to the RNA binding groove (Fig. 4A).

In EBOV NP core, the hydrophobic pocket is comprised by Helix-14, Helix-15, and Helix-19, a number of hydrophobic residues contributed for the formation of this pocket, including V247, V250, 1254, L255, L264, A268, V273, V277, F280, L284, L287, and L316 (Figs. 1D and 4C). However, 
this hydrophobic pocket in occupied by $\mathrm{P}$ protein in NiV NP, but is accommodated by Helix-20, which is consisted by

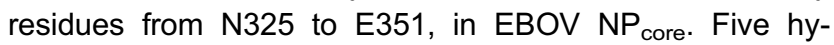
drophobic residues, i.e. V336, Y340, L343, A346, and A347, stabilize the interaction between Helix-20 with the hydrophobic pocket in C-lobe.

We next investigated the conservation of this hydrophobic pocket among filoviridae family. Five strains of Ebola virus (strain Zaire, UniProt 072142; Sudan, UniProt Q5XX08; Bundibugyo, UniProt B8XCM7; Tai forest, UniProt B8XCN6; and Reston, UniProt Q8JPY1), five strains of Marburg virus (strain Angola/2005, UniProt Q1PD53; Ozolin/1975, UniProt Q6UY69; Popp/1967, UniProt P35263; Ravn/1987, UniProt Q1PDD0; and Musoke/1980, UniProt P27588) and one strain of Lloviu cuevavirus (strain Asturias-Bat86/2003, UniProt G8EFI1) are aligned (Fig. 4D). All of the hydrophobic residues consisting of the pocket are highly-conservative among all the given strains, suggesting the conserved structural architecture of RNP formation among different genus of this family and the critical importance of the function of this hydrophobic pocket.

\section{DISCUSSION}

Structural studies in the past ten years have led an understanding of -ssRNA virus-encoded NPs (Sun et al., 2012; Zhou et al., 2013). Although these NPs possess large variations, their structures can be divided into two topological groups. The first group includes most -ssRNA viral NPs, including BDV from the Bornaviridae family (Rudolph et al., 2003), VSV and rabies virus from the Phabdoviridae family (Albertini et al., 2006; Green et al., 2006), RSV from the Paramyxoviridae family (Tawar et al., 2009), influenza virus from the Orthomyxoviridae family (Ye et al., 2006), Rift Valley fever virus (RVFV) (Phlebovirus genus) (Raymond et al., 2010), and Bunyamwera virus (BUNV) (Orthobunyavirus

A

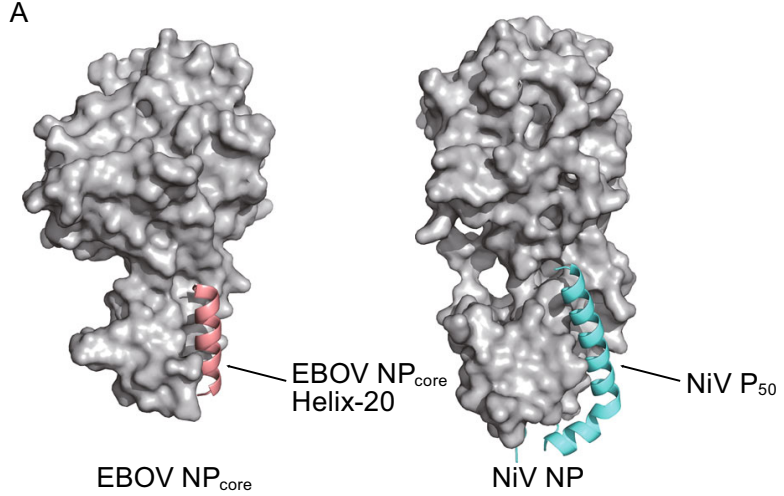

B

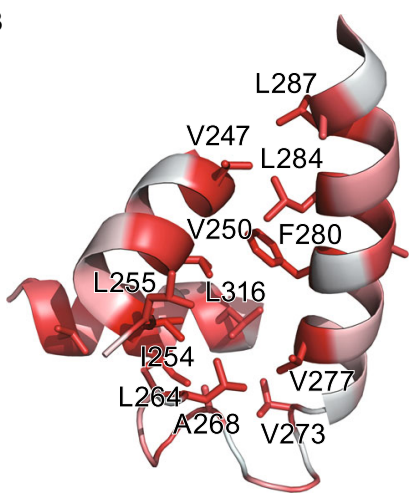

C

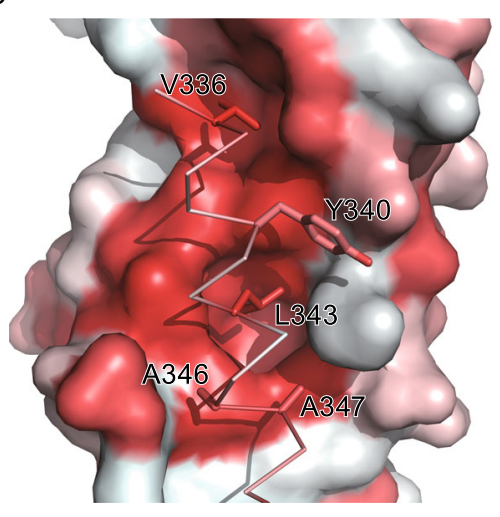

D

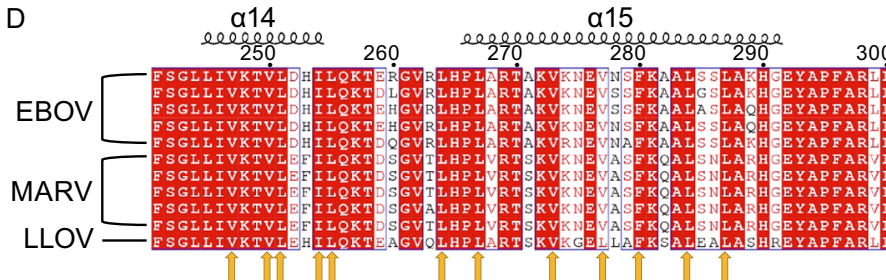

$$
\alpha 16 \alpha 17 \propto 18 \alpha 19
$$

$\alpha 20$

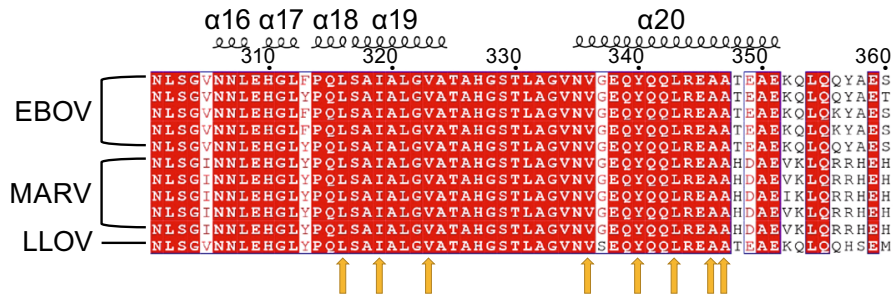

Figure 4. Helix-20 in EBOV NP core indicates an essential hydrophobic pocket. (A) Comparison of EBOV NP Both structures are shown as surface and colored gray. EBOV NP $P_{\text {core }}$ Helix-20 and NiV $\mathrm{P}_{50}$ are shown as cartoon, colored salmon red and cyan, respectively. (B) Formation of the hydrophobic pocket. Main chain is shown in cartoon and hydrophobic residues are shown in stick, colored in red by hydrophobicity. (C) Interaction of Helix-20 with hydrophobic pocket. Hydrophobic pocket are shown in surface, Helix-20 are displayed in ribbon and stick, colored in red by hydrophobicity. (D) Primary sequence alignment of members of the filoviridae family. Highly conserved residue in Helix-14, Helix-15, Helix-19, and Helix-20 are indicated by yellow arrow. 
genus) (Li et al., 2013) within the Bunyaviridae family. Although their detailed structures are different, NPs in class I possess a general $\mathrm{N}$ - and $\mathrm{C}$-lobe that face each other to form a positively charged crevice for RNA binding but use diverse structural components for the inter-protomer interaction (Sun et al., 2012). NPs in class II include LAFV (Arenaviridae family) (Hastie et al., 2011a, b; Qi et al., 2011) and CCHFV (Nairovirus genus, Bunyaviridae family) (Guo et al., 2012). Although LAFV and CCHFV belong to different virus families and their NPs were found to have additional biological functions, the structural regions for genome encapsidation are highly similar. According to the structural topology, the structure of EBOV NP core belongs to class I viral NP. However, the extension from the core domain to the end of EBOV NP, including a non-conservative region and the C-terminal minor domain which is of about two hundred amino-acid length, distinguishes EBOV NP with other class I viral NPs. Actually, the primary sequence of Filoviridae shares high similarity with Paramyxoviridae family in the first 450 amino acids, but shows its own specificity for the $\mathrm{C}$-terminal region. The precise biological function of this extension part warrant further investigations.

Although NP is known to be the most, at least one of the most, stable proteins encoded by -ssRNA virus during their proliferation cycle, significant structural shift can be observed in various stages among multiple virus species (Zhou et al., 2013). The most essential results were acquired from the structures of phlebovirus-encoded NPs. The first RVFV NP purified through a denature/refolding method in monomeric form shows a novel compact structure that lacks a positively charged crevice for RNA binding and has no protruding portions for NP oligomerization (Raymond et al., 2010). However, Ferron et al. used a different purification method in physiologically condition to solve the hexameric structure of RVFV NP, which has a highly positively charged inner perimeter as the RNA-binding site (Ferron et al., 2011). Although the body regions of the two structures obtained through different purification methods are identical, significant conformational difference occurs at the $\mathrm{N}$-terminal arm ( $\mathrm{N}$-arm). The distinct positions of the $\mathrm{N}$-arm reflect the structural flexibility during RNP formation, in which the monomeric structure may represent a "waiting" conformation before oligomerization and binding with RNA (Ferron et al., 2011). Subsequently, Raymond et al. reported the structures of the NP-RNA complexes of the RVFV and Toscana viruses, other members of the Phlebovirus genus, in tetrameric, pentameric, and hexameric forms (Fig. 3D) (Raymond et al., 2012). These structures confirmed that the highly flexible $\mathrm{N}$-arm mediates the contacts between NP protomers, which are responsible for RNP formation (Raymond et al., 2012). This monomeric building block and the flexibility of the NP-NP interaction in the oligomer formation allow RVFV RNP to pack into viral particles with higher structures and density (Raymond et al., 2012). All these results revealed that the component of viral NP could have structural shift in the different stage of virus life cycle to facilitate either RNA binding or further oligomerization to form RNP. During the preparation of this manuscript, Daisy W. Leung et al. reported an EBOV NP structure complex with a peptide from VP35 at 3.7- $\AA$ resolution. These two EBOV NP structures may present another essential instance for conformation change during virus life cycle (Fig. 5). In the structure of EBOV NP core alone reported in this work, Helix-20 folds towards and interacts with the hydrophobic pocket on the interface of the C-lobe of EBOV NP core $_{\text {to form a compact }}$ structure. In contrast, in the structure of EBOV NP core in complex with VP35 peptide, Helix-20, together with Helix-21, transits to the opposite side of the C-lobe of EBOV NP core (Leung et al., 2015) (Fig. 5B). Because EBOV VP35 peptide (NPBP, residues 20-48) binds NP with high affinity and specificity, inhibits NP oligomerization, and releases RNA from NP-RNA complexes in vitro, this structure is likely to represent a transition state immediately before the initiation of viral RNA synthesis in RdRp. We would like to propose that EBOV NP core structure reported in our work is the original state of EBOV NP after it can be translated from host ribosome, which keeps in $\mathrm{N}^{0}$ stage, ready for the binding with nascent RNA and the formation of RNP.

Actually, the transition state is very similar to the function of a phosphoprotein $(P)$ in VSV replication (Green and Luo, 2009). $P$ protein is an essential co-factor of VSV RNP. In the structure of VSV RNP-like particle (NP-RNA complex) in complex with $\mathrm{C}$-terminal domain of $\mathrm{P}, \mathrm{P}$ binds primarily to the C-terminal lobe of two adjacent $N$ proteins within nucleocapsid, and the proximity to RNA cavity indicates that the $\mathrm{P}$ protein binding impacts the RNA encapsidation of VSV NP and orients the $L$ protein to access the RNA template without NP (Green and Luo, 2009). Although both P of VSV and VP35-NPBP of EBOV can regulate RNA encapsidation by NPs, significant variations still distinguish them. First, VSV NP can still bind with RNA, even though P protein binds to VSV RNP-like particle, indicating suggesting that the interaction of $P$ with NP cannot directly induce the releasing of RNA in VSV. However, EBOV VP35-NPBP can directly and competitively inhibit the binding of RNA to EBOV NP. Moreover, there is no obvious structural shift of NP component in VSV during $P$ protein binding, but Helix-20 in EBOV NP has significant remodeling with VP35-NPBP interaction. All these differences suggest the potential existence of several other architecture(s) from the original state of EBOV NP to the transition state before RNA synthesis. This warrants further structural and biological validations, such as, the complex structure of EBOV nucleoprotein bound with RNA.

Because the correct RNP formation and function is a key step for the replication, transcription, and assembly for -ssRNA viruses, it is conceivable that blockage of this process would provide great potential for antiviral development. In the last few years, great progress has been achieved based on this new antiviral strategy, particularly for the influenza virus. Through a chemical genetic method, Kao et al. first identified that influenza virus NP is a druggable target 
A
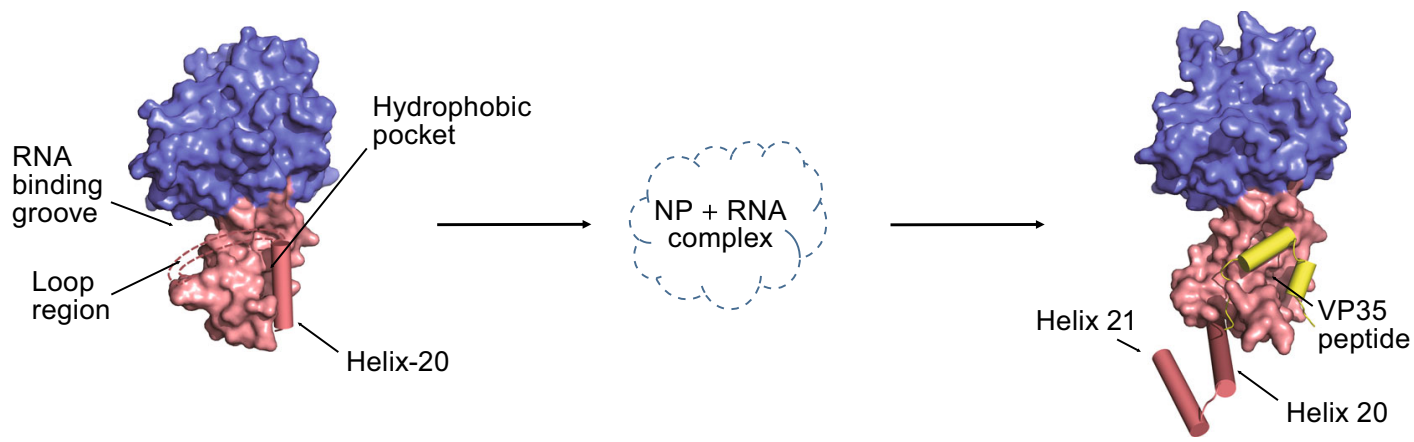

B

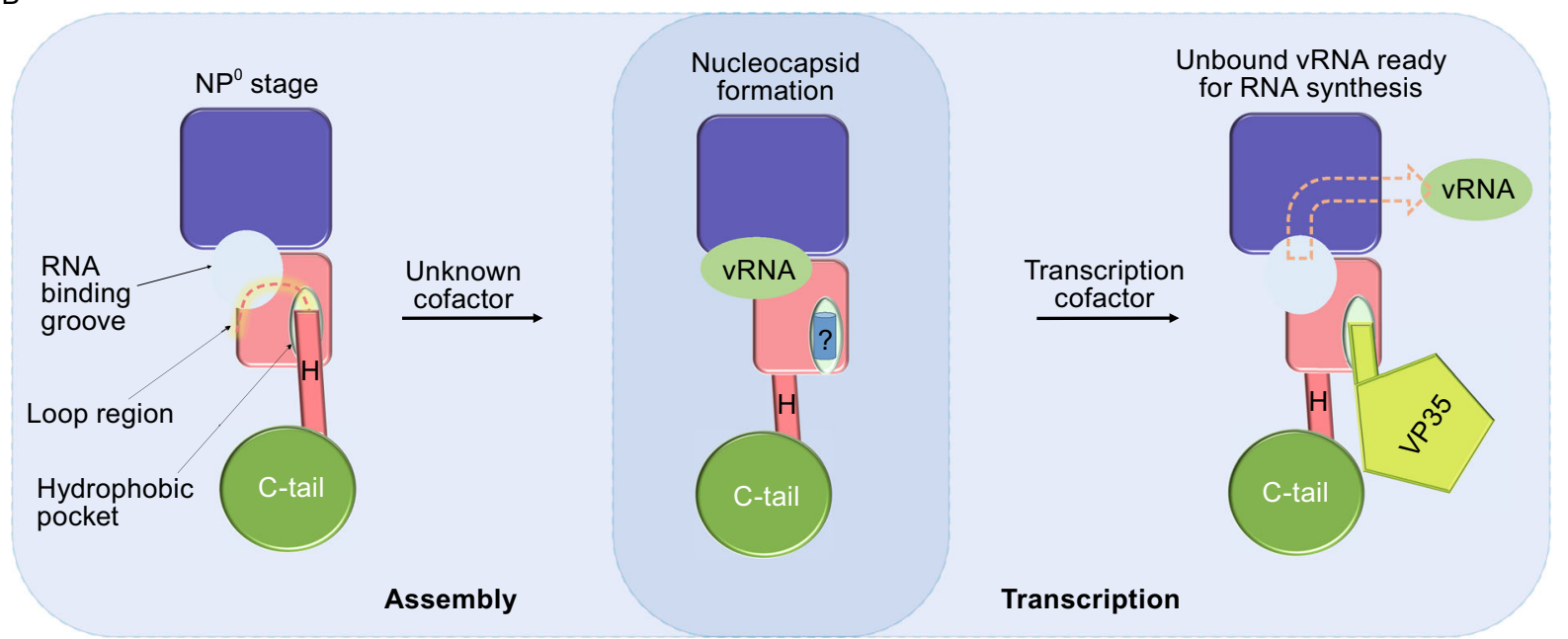

Figure 5. Structural mobility of EBOV NP core within virus proliferation. (A) Structural comparison of EBOV NP with VP35 peptide. The molecule of EBOV NP core alone and in complex with VP35 peptide are shown in the left and right panels. The $\mathrm{N}$-lobe and C-lobe are colored as blue and red, respectively. Helices 20-21 are shown as cylinder, and the loop region (A326-V334) are linked by dotted lines. Small peptide derived from VP35 is shown as yellow cylinders. (B) A proposed model of conformation change during nucleocapsid assembly and transcription process. RNA binding groove and hydrophobic pocket are indicated by arrow.

(Kao et al., 2010). They reported that nucleozin, a small molecule compound that triggers aggregation and inhibits the nuclear accumulation of NP, can inhibit the replication of influenza virus at a nanomolar median effective concentration $\left(E_{50}\right.$ ) (Kao et al., 2010). In a parallel effort, Gerritz et al. discovered a series of influenza replication inhibitors and showed that they interfere with NP-dependent processes via the formation of higher-order NP oligomers with an $\mathrm{EC}_{50}$ up to $60 \mathrm{nmol} / \mathrm{L}$ (Gerritz et al., 2011). Notably, the structure of NP in complex with a representative compound of these inhibitors revealed that two inhibitors in an antiparallel orientation lock two adjacent NP protomers. This unexpected quaternary complex explained viral inhibition via the ligandinduced formation of stable NP oligomers (Gerritz et al., 2011). These results cumulatively demonstrated that targeting the formation of viral RNP is a valid goal for the development of small-molecule therapies against viral resistance to currently available drugs targeting surface protein. Here we report a distinct hydrophobic pocket in this work, which shows highly conservative among all viruses within Filoviridae. Therefore, the structure of EBOV NP not only aids in understanding the structural and functional differences among NPs encoded by -ssRNA viruses, but also benefits the development of antiviral therapies against EBOV infection.

\section{MATERIALS AND METHODS}

\section{Protein production}

The gene of the Zaire ebolavirus nucleoprotein (residues 36-351) was cloned into the pET-21d expression vector within Ncol and Xhol site following a general protocol, The sequences of the primers are: forward, 5'-CCCATGGCTGTTCGGCAAAGAGTCATC-3', reverse, 5'-CCCTCGAGCTCAGCCTCAGTGGCAGCCTC-3'. The accuracy of the inserts was verified by sequencing.

The recombinant plasmid of EBOV NP core was transformed into E. coli strain BL21 (DE3) and overexpressed as a $6 \times$ His tag fused at the $\mathrm{C}$ terminus fusion protein. The cells were cultured at $37^{\circ} \mathrm{C}$ in 
$800 \mathrm{~mL}$ LB media containing $100 \mu \mathrm{g} / \mathrm{mL}$ ampicillin. Once $\mathrm{OD}_{600}$ reached 0.6 , the culture was transferred to $16^{\circ} \mathrm{C}$, and protein was induced by incubating with $0.25 \mathrm{mmol} / \mathrm{L}$ isopropyl- $\beta-\mathrm{D}-1$-thiogalactopyranoside (IPTG) for an additional $18 \mathrm{~h}$. Harvested cells were resuspended in lysis buffer $(20 \mathrm{mmol} / \mathrm{L}$ Tris- $\mathrm{HCl}, 500 \mathrm{mmol} / \mathrm{L} \mathrm{NaCl}$, $\mathrm{pH}$ 8.5) and homogenized with a low-temperature ultra-high pressure cell disrupter (JNBIO, China). The lysate was centrifuged at $25,000 \times g$ for $30 \mathrm{~min}$ at $4^{\circ} \mathrm{C}$ to remove cell debris. The supernatant was then loaded twice onto a Ni-NTA column pre-equilibrated with lysis buffer. Resin was washed four times with $60 \mathrm{~mL}$ of wash buffer (20 mmol/L Tris- $\mathrm{HCl}, 500 \mathrm{mmol} / \mathrm{L} \mathrm{NaCl}, 25 \mathrm{mmol} / \mathrm{L}$ imidazole, $\mathrm{pH}$ 8.5) and eluted with $30 \mathrm{~mL}$ of wash buffer supplemented with $1 \mathrm{~mol} / \mathrm{L}$ imidazole. The protein was further purified on a Superdex-200 (GE Healthcare) column equilibrated with the buffer containing $20 \mathrm{mmol} / \mathrm{L}$ Tris-HCl, $200 \mathrm{mmol} / \mathrm{L} \mathrm{NaCl}, 5 \mathrm{mmol} / \mathrm{L}$ DTT, pH 8.5. SDS-PAGE analysis revealed over $95 \%$ purity of the final purified recombinant protein. Fractions from the single major peak were pooled and concentrated to $6 \mathrm{mg} / \mathrm{mL}$ for crystallization. The purified EBOV NP core was $>95 \%$ pure according to SDS-PAGE analysis and had an $A_{280} / A_{260}$ ratio of 1.6. The purified protein was concentrated to $10 \mathrm{mg} / \mathrm{mL}$ and stored at 193 K.

Selenomethionine (SeMet) derivative of EBOV NP core was produced in the methionine-auxotrophic E. coli strain B834 (DE3) that was grown in minimal medium supplemented with $3 \%$ glucose, $30 \mathrm{mg} / \mathrm{L} \mathrm{L}$-selenomethionine, and $100 \mu \mathrm{g} / \mathrm{mL}$ ampicillin. When $\mathrm{OD}_{600}$ reached 0.6 , the culture was transferred to $16^{\circ} \mathrm{C}$, another $30 \mathrm{mg} / \mathrm{L} \mathrm{L}-$ selenomethionine was added in. Protein was induced by incubating with $0.25 \mathrm{mmol} / \mathrm{L}$ IPTG for an additional $24 \mathrm{~h}$. SeMet sub-

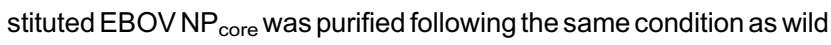
type protein.

\section{Crystallization}

Initial crystallization trials were performed in a 96-well format using a $1: 1$ ratio of well solution to protein at $5.5 \mathrm{mg} / \mathrm{mL}$ by screening commercial crystal screening kits at $16^{\circ} \mathrm{C}$, including the Index, Crystal Screen, PEG/lon, Salt/RX, Natrix, Crystal Screen Lite, and Crystal Screen cryo from Hampton Research. Small crystals of

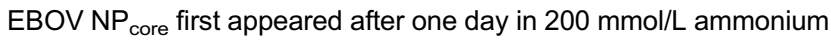
citrate tribasic $\mathrm{pH} 7.0$ and $20 \%(w / v)$ PEG3350.

Further optimization with additive and detergent screens (Hampton Research) was performed, the final optimized crystal condition was $200 \mathrm{mmol} / \mathrm{L}$ ammonium citrate tribasic $\mathrm{pH} 7.5$ and $18 \%(w / v)$ PEG3350. Cubic-like crystals grew to a final size of $40 \mu \mathrm{m}$ $\times 40 \mu \mathrm{m} \times 60 \mu \mathrm{m}$ within three days at $16^{\circ} \mathrm{C}$. Crystals were harvested and protected in the well solution containing $30 \%$ (w/v) PEG3350 and cooled in dry nitrogen stream at $100 \mathrm{~K}$ for X-ray data collection. SeMet EBOV NP core crystals were grown in the same condition.

X-ray data collection, processing, and structure determination

All crystals were gradually transferred into a harvesting solution containing the respective precipitant solutions plus $5 \%(\mathrm{v} / \mathrm{v})$ glycerol before being flash frozen in liquid nitrogen for storage. Data were collected under cryogenic conditions at $100 \mathrm{~K}$. The selenomethionine SAD data set of the EBOV NP core was collected at $2.4 \AA$ using a wavelength corresponding to the Se peak at the SSRF (Shanghai,
China) beamline BL19U, and another native data set was collected at $1.8 \AA$. All data sets were processed using the HKL-3000 package (Minor et al., 2006). The crystals belonged to the space group $P 2{ }_{1} 2{ }_{1} 2$ with cell parameters $a=59.8 \AA, b=162.9 \AA, c=31.3 \AA$, and $\alpha=\beta=\gamma=90^{\circ}$. Excluding the first selenium of each polypeptide, 6 of 7 selenium atoms in the asymmetric unit were located and refined, and the SAD data phases were calculated and substantially improved by solvent flattening using the PHENIX program (Adams et al., 2002). A model was manually built into the modified experimental electron density using COOT (Emsley and Cowtan, 2004) and further refined in PHENIX. Model geometry was verified using the program MolProbity (Lovell et al., 2003). The final refinement statistics are summarized in Table 1. Structural figures were drawn using the program PyMOL (DeLano, 2002).

\section{Accession code}

The coordinates and structure factors have been deposited with the RCSB under accession codes: 4Z9P. The authors declare no competing financial interest.

\section{ACKNOWLEDGMENTS}

We gratefully acknowledge Dr. RongGuang Zhang and other staffs of BL19U, SSRF, China for their assistance with the diffraction data collection. This work was supported by the National Basic Research Program (973 Program) (Nos. 2014CB542800 and 2013CB911103), National Major Scientific and Technological Special Project for "Significant New Drugs Development" (Grant No. 2013ZX09301306), the National Natural Science Foundation of China (Grant Nos. 31300606 and 81322023) and Tianjin Municipal Natural Science Foundation (Grant No. 14JCQNJC10100).

\section{ABBREVIATIONS}

-ssRNA, negative-sense single-stranded RNA; BDV, Borna disease virus; BUNV, Bunyamwera virus; cRNA, complementary positivestrand RNA; CCHFV, Crimean-Congo hemorrhagic fever; EBOV, Ebola virus; GP, glycoprotein; LASV, Lassa fever virus; LLOV, Lloviu virus; MARV, Marburg virus; NP, nucleocapsid protein; RdRp, RNAdependent RNA polymerase; RNP, ribonucleoprotein; RSV, respiratory syncytial virus; RVFV, Rift Valley fever virus; VP, viral protein; VSV, vesicular stomatitis virus.

\section{COMPLIANCE WITH ETHICS GUIDELINES}

Shishang Dong, Peng Yang, Guobang Li, Baocheng Liu, Wenming Wang, Xiang Liu, Boran Xia, Cheng Yang, Zhiyong Lou, Yu Guo and Zihe Rao declare that they have no conflict of interest.

This article does not contain any studies with human or animal subjects performed by the any of the authors.

\section{OPEN ACCESS}

This article is distributed under the terms of the Creative Commons Attribution 4.0 International License (http://creativecommons.org/ licenses/by/4.0/), which permits unrestricted use, distribution, and reproduction in any medium, provided you give appropriate credit to 
the original author(s) and the source, provide a link to the Creative Commons license, and indicate if changes were made.

\section{REFERENCES}

Adams PD et al (2002) PHENIX: building new software for automated crystallographic structure determination. Acta Crystallogr D Biol Crystallogr 58(Pt 11):1948-1954

Albertini AA et al (2006) Crystal structure of the rabies virus nucleoprotein-RNA complex. Science 313(5785):360-363

Ariza A et al (2013) Nucleocapsid protein structures from orthobunyaviruses reveal insight into ribonucleoprotein architecture and RNA polymerization. Nucleic Acids Res 41(11):5912-5926

Arranz R et al (2013) The structure of native influenza virion ribonucleoproteins. Science 338(6114):1634-1637

Bharat TA et al (2011) Cryo-electron tomography of Marburg virus particles and their morphogenesis within infected cells. PLoS Biol 9(11):e1001196

Bharat TA et al (2012) Structural dissection of Ebola virus and its assembly determinants using cryo-electron tomography. Proc Natl Acad Sci USA 109(11):4275-4280

Chenavas S et al (2013) Monomeric nucleoprotein of influenza a virus. PLoS Pathog 9(3):e1003275

DeLano W (2002) The PyMOL Molecular Graphics System 2002

Dong $\mathrm{H}$ et al (2013) Structure of Schmallenberg orthobunyavirus nucleoprotein suggests a novel mechanism of genome encapsidation. J Virol 87(10):5593-5601

Dziubanska PJ et al (2014) The structure of the C-terminal domain of the Zaire ebolavirus nucleoprotein. Acta Crystallogr D Biol Crystallogr 70(Pt 9):2420-2429

Emsley P, Cowtan K (2004) Coot: model-building tools for molecular graphics. Acta Crystallogr D Biol Crystallogr 60(Pt 12 Pt 1):21262132

Ferron F et al (2011) The hexamer structure of Rift Valley fever virus nucleoprotein suggests a mechanism for its assembly into ribonucleoprotein complexes. PLoS Pathog 7(5):e1002030

Gerritz SW et al (2011) Inhibition of influenza virus replication via small molecules that induce the formation of higher-order nucleoprotein oligomers. Proc Natl Acad Sci USA 108 (37):15366-15371

Green TJ, Luo M (2009) Structure of the vesicular stomatitis virus nucleocapsid in complex with the nucleocapsid-binding domain of the small polymerase cofactor, P. Proc Natl Acad Sci USA 106 (28):11713-11718

Green TJ et al (2006) Structure of the vesicular stomatitis virus nucleoprotein-RNA complex. Science 313(5785):357-360

Guo $Y$ et al (2012) Crimean-Congo hemorrhagic fever virus nucleoprotein reveals endonuclease activity in bunyaviruses. Proc Natl Acad Sci USA 109(13):5046-5051

Hastie KM et al (2011a) Structure of the Lassa virus nucleoprotein reveals a dsRNA-specific 3' to 5' exonuclease activity essential for immune suppression. Proc Natl Acad Sci USA 108(6):23962401

Hastie KM et al (2011b) Crystal structure of the Lassa virus nucleoprotein-RNA complex reveals a gating mechanism for RNA binding. Proc Natl Acad Sci USA 108(48):19365-19370
Holm L, Rosenstrom P (2010) Dali server: conservation mapping in 3D. Nucl Acids Res 38:W545-W549

Huang $Y$ et al (2002) The assembly of Ebola virus nucleocapsid requires virion-associated proteins 35 and 24 and posttranslational modification of nucleoprotein. Mol Cell 10(2):307-316

Jiao L et al (2013) Structure of severe Fever with thrombocytopenia syndrome virus nucleocapsid protein in complex with suramin reveals therapeutic potential. J Virol 87(12):6829-6839

Kao RY et al (2010) Identification of influenza A nucleoprotein as an antiviral target. Nat Biotechnol 28(6):600-605

Kranzusch PJ, Whelan SP (2011) Arenavirus Z protein controls viral RNA synthesis by locking a polymerase-promoter complex. Proc Natl Acad Sci USA 108(49):19743-19748

Krissinel E, Henrick K (2004) Secondary-structure matching (SSM), a new tool for fast protein structure alignment in three dimensions. Acta Crystallogr D Biol Crystallogr 60(Pt 12 Pt 1):22562268

Kuhn JH et al (2010) Proposal for a revised taxonomy of the family Filoviridae: classification, names of taxa and viruses, and virus abbreviations. Arch Virol 155(12):2083-2103

Leung DW et al (2015) An intrinsically disordered peptide from Ebola Virus VP35 controls viral RNA synthesis by modulating nucleoprotein-RNA interactions. Cell Rep 11:1-14

$\mathrm{Li} B$ et al (2013) Bunyamwera virus possesses a distinct nucleocapsid protein to facilitate genome encapsidation. Proc Natl Acad Sci USA 110(22):9048-9053

Lovell SC et al (2003) Structure validation by Calpha geometry: phi, psi and Cbeta deviation. Proteins 50(3):437-450

Minor W et al (2006) HKL-3000: the integration of data reduction and structure solution-from diffraction images to an initial model in minutes. Acta Crystallogr D Biol Crystallogr 62(Pt 8):859-866

Moeller $A$ et al (2013) Organization of the influenza virus replication machinery. Science 338(6114):1631-1634

Muhlberger $\mathrm{E}$ et al (1999) Comparison of the transcription and replication strategies of marburg virus and Ebola virus by using artificial replication systems. J Virol 73(3):2333-2342

$\mathrm{Ng}$ AK et al (2008) Structure of the influenza virus A H5N1 nucleoprotein: implications for RNA binding, oligomerization, and vaccine design. Faseb J 22(10):3638-3647

Niu $F$ et al (2013) Structure of the Leanyer orthobunyavirus nucleoprotein-RNA complex reveals unique architecture for RNA encapsidation. Proc Natl Acad Sci USA 110(22):9054-9059

Noda T et al (2010) Characterization of the Ebola virus nucleoprotein-RNA complex. J Gen Virol 91(Pt 6):1478-1483

Qi X et al (2011) Cap binding and immune evasion revealed by Lassa nucleoprotein structure. Nature 468(7325):779-783

Raymond DD et al (2010) Structure of the Rift Valley fever virus nucleocapsid protein reveals another architecture for RNA encapsidation. Proc Natl Acad Sci USA 107(26):11769-11774

Raymond DD et al (2012) Phleboviruses encapsidate their genomes by sequestering RNA bases. Proc Natl Acad Sci USA 109 (47):19208-19213

Reguera J et al (2013) Structural basis for encapsidation of genomic RNA by La Crosse Orthobunyavirus nucleoprotein. Proc Natl Acad Sci USA 110(18):7246-7251

Rudolph MG et al (2003) Crystal structure of the borna disease virus nucleoprotein. Structure 11(10):1219-1226 
Ruigrok RW, Crepin T, Kolakofsky D (2011) Nucleoproteins and nucleocapsids of negative-strand RNA viruses. Curr Opin Microbiol 14(4):504-510

Sun Y, Guo Y, Lou Z (2012) A versatile building block: the structures and functions of negative-sense single-stranded RNA virus nucleocapsid proteins. Protein Cell 3(12):893-902

Tawar RG et al (2009) Crystal structure of a nucleocapsid-like nucleoprotein-RNA complex of respiratory syncytial virus. Science 326(5957):1279-1283

Watanabe S, Noda T, Kawaoka Y (2006) Functional mapping of the nucleoprotein of Ebola virus. J Virol 80(8):3743-3751
Yabukarski F et al (2014) Structure of Nipah virus unassembled nucleoprotein in complex with its viral chaperone. Nat Struct Mol Biol 21(9):754-759

Ye Q, Krug RM, Tao YJ (2006) The mechanism by which influenza A virus nucleoprotein forms oligomers and binds RNA. Nature 444 (7122):1078-1082

Zhou $\mathrm{H}$ et al (2013) Structural perspective on the formation of ribonucleoprotein complex in negative-sense single-stranded RNA viruses. Trends Microbiol 21(9):475-484 